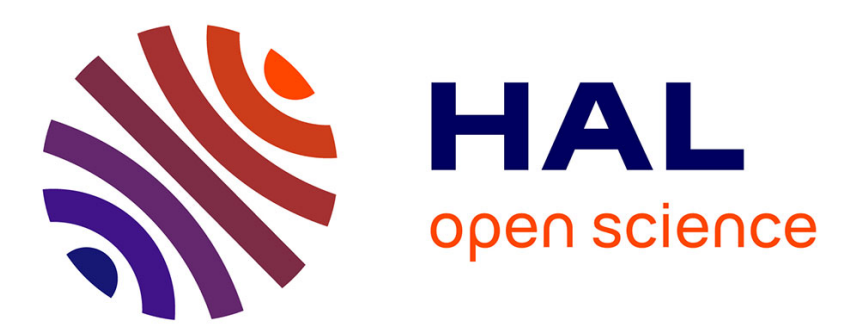

\title{
Efficient Cooperative Protocols for General Outage-Limited Multihop Wireless Networks
}

Behrouz Maham, Walid Saad, Merouane Debbah, Zhu Han, Are Hjorungnes

\section{To cite this version:}

Behrouz Maham, Walid Saad, Merouane Debbah, Zhu Han, Are Hjorungnes. Efficient Cooperative Protocols for General Outage-Limited Multihop Wireless Networks. PIMRC 2010, Sep 2010, Turkey. 6 p. hal-00556158

\section{HAL Id: hal-00556158 \\ https://hal-centralesupelec.archives-ouvertes.fr/hal-00556158}

Submitted on 15 Jan 2011

HAL is a multi-disciplinary open access archive for the deposit and dissemination of scientific research documents, whether they are published or not. The documents may come from teaching and research institutions in France or abroad, or from public or private research centers.
L'archive ouverte pluridisciplinaire HAL, est destinée au dépôt et à la diffusion de documents scientifiques de niveau recherche, publiés ou non, émanant des établissements d'enseignement et de recherche français ou étrangers, des laboratoires publics ou privés. 


\title{
Efficient Cooperative Protocols for General Outage-Limited Multihop Wireless Networks
}

\author{
Behrouz Maham $^{\dagger}$, Walid Saad ${ }^{\dagger}$, Mérouane Debbah ${ }^{\ddagger}, Z^{2}$ u Han ${ }^{\S}$, and Are Hjørungnes ${ }^{\dagger}$ \\ $\dagger$ UNIK - University Graduate Center, University of Oslo, Norway \\ ‡Alcatel-Lucent Chair on Flexible Radio, SUPÉLEC, Gif-sur-Yvette, France \\ ${ }^{\S}$ ECE Department, University of Houston, USA \\ Email: behrouzmeifi.uio.no, saadeunik.no, merouane.debbahesupelec.fr, \\ zhan2@mail.uh. edu, arehj@unik. no
}

\begin{abstract}
Due to the limited energy supplies of nodes in wireless networks, achieving energy efficiency is crucial for extending the lifetime of these networks. Thus, we study efficient power allocations and transmission protocols for outage-restricted multihop wireless networks based on cooperative transmission. In such multihop networks, a number of nodes, acting as relays, can assist a source node in the transmission of its messages to a single destination. In this paper, several multihop transmission protocols with cooperative routing are proposed. Each of the proposed protocols offers a different rate and energy efficiency. Cooperative routing protocols are introduced using arbitrary distributed space-time codes for the purpose of energy savings, given a required outage probability at the destination. Three efficient cooperative multihop transmissions are proposed, and their corresponding distributed power allocation schemes, which depend only on the statistics of the channels, are also derived. The proposed cooperative protocols offer different degrees of energy efficiency, spectral efficiency, complexity and signalling overhead. Simulations show that, using the proposed cooperative protocols, substantial energy savings are achievable, compared to non-cooperative multihop routing, in a network having an outage probability constraint.
\end{abstract}

\section{INTRODUCTION}

Energy consumption in multihop wireless networks is a crucial issue that needs to be addressed at all the communication layers, from the hardware up to the application. The focus of this paper is on energy efficiency when messages may be transmitted via multiple radio hops. After substantial research efforts in the last several years, routing for multihop wireless networks is a broadly investigated problem [1], [2]. Nevertheless, with the emergence of new multiple-antennas technologies, existing routing solutions in the traditional radio transmission model are no longer efficient. For instance, it is feasible to coordinate the simultaneous transmissions from multiple transmitters to one receiver simultaneously. As a result, simultaneous transmitter signals from several different nodes to the same receiver are not considered a collision, but instead could be combined at the receiver to obtain stronger signal. The routing problem in the cooperative radio transmission model over static channels is studied in [3], where it is allowed that multiple nodes along a path coordinate together to transmit a message to the next hop as long as the

This work was supported by the Research Council of Norway through the project 176773/S10 entitled "Optimized Heterogeneous Multiuser MIMO Networks - OptiMO" and the project 183311/S10 entitled "Mobile-to-Mobile Communication Systems (M2M)". combined signal at the receiver satisfies a given SNR threshold value. In [3], it is assumed that transmitting nodes adjust their phases in such a way that the coherent reception of signals at the receiving node is possible. However, the knowledge of the instantaneous channels at the transmitting nodes is difficult to realize. The cooperative routing problem has been further considered in the literature [4], [5]. In [4], a cooperative-based routing algorithm, namely, the Minimum Power Cooperative Routing (MPCR) algorithm is proposed, which makes use of the incremental relaying while constructing the minimumpower route. In [6], two heuristic routing algorithms, namely, Cooperative routing along Truncated Non-Cooperative Route (CTNCR) and Source Node Expansion Routing (SNER) are proposed. These algorithms choose the minimum-power route while guaranteeing fixed transmission rate. In [7], Maham et al. proposed a cooperative multihop routing for the purpose of power savings, constrained on a required bit error rate (BER) at the destination. In [5], Pandana et al. studied the impact of cooperative communication on maximizing the lifetime of wireless sensor networks. Further, the work in [8] studies the tradeoff between delay and bit error rate in cooperative multihop routing using game theory. However, in all of the cooperating routings stated above, the knowledge of channel is required at transmitters, which makes the implementation difficult. In [9], a new cooperative routing protocol is introduced using the Alamouti space-time code for the purpose of energy savings, given a required outage probability at the destination. However, since Alamouti code is used, the number of cooperative nodes is limited to two.

In this paper, a cooperative multihop routing scheme is proposed for Rayleigh fading channels. The investigated system can achieve considerable energy savings compared to noncooperative multihop transmission, when there is an outage probability quality-of-service $(\mathrm{QoS})$ requirement at the destination node. Three efficient cooperative protocols are proposed for multihop networks that highlight a tradeoff among energy efficiency, throughput, and complexity, based on the topology of network. Moreover, distributed power control schemes are derived to minimize the total transmission power given the outage probability constraint. Using some tight approximations, simple closed-form power allocations are presented without requiring the knowledge of instantaneous channel state information (CSI); hence, the proposed schemes can be imple- 
mented in real wireless systems. Numerical results show that the proposed power allocation strategies provide considerable gains compared to non-cooperative multihop transmission.

\section{System Model}

Consider a wireless communication scenario where a source node $s$ transmits information to a destination node $d$ with the assistance of $N$ intermediate relays. Due to the broadcast nature of the wireless channel, some relays can overhear the transmitted information, and, thus, can cooperate with the source to send its data. The wireless link between any two nodes in the network is modeled as a Rayleigh fading narrowband channel. For medium access, the relays are assumed to transmit over orthogonal channels, thus, no interrelay interference is considered in the signal model.

Similar to [3], each transmission is either a broadcast transmission whereby a single node transmits the information that is received by multiple nodes, or a cooperative transmission whereby multiple nodes simultaneously send the information to a single receiver. Another important assumption is on synchronization among nodes. There are three levels of synchronization: frame, symbol, and carrier. We assume that the receivers are completely synchronized at all levels. For transmitters, it is realistic to assume that frame- and symbollevel synchronization are available. The contentious point is on carrier-level synchronization, which requires that separate microwave oscillators at different nodes are synchronized. This seems highly unrealistic. Left by themselves, the drift of the oscillators makes synchronization difficult. We will therefore assume that there is no carrier synchronization, and the power allocations studied in this paper are independent of the knowledge of channel phases. However, recently in [10], some efficient carrier synchronization techniques are introduced enable transmit beamforming. In addition, using nontrivial microwave innovations, it might be possible to couple oscillators, and, also, very closely spaced nodes could even auto-couple.

The transmission protocols have two main stages: Noncooperative transmission and space-time cooperative transmission. Depending on the protocol, non-cooperative stage may consist of one are multiple phases. The subsequent phases use space-time cooperation scheme. The detailed description of our proposed protocols will be given in the next section. Assume the source intends to transmit $K$ symbol (or packet) in time $t$ toward the destination. The signals transmitted by the source terminal during the $k$ th time slot of Phase 1 is denoted as $s_{k}(t), k=1, \ldots, K$. In the following, symbol-by-symbol transmission (or packet-by-packet) is considered such that the time index $t$ can be dropped; hence, $s_{k}, k=1, \ldots, K$ is the signal transmitted in the $k$ th time slot, where $\mathbb{E}\left\{s_{k}\right\}=0$ and $\mathbb{E}\left\{\left|s_{k}\right|^{2}\right\}=1$ for $k=1, \ldots, K$. The data symbols may be chosen from a complex- valued finite constellation such as quadrature amplitude modulation (QAM) or from a Gaussian codebook. In Phase 1, the source transmits the information, and the signal received at the $i$ th node in the first $K$ time slots is given by

$$
y_{i, k}=\sqrt{P_{0,1}} h_{0, i} s_{k}+v_{i, k}, \text { for } k=1, \ldots, K,
$$

where $P_{0,1}$ is the average transmitted symbol energy of the source during the first phase (since the information symbols $s_{k}$ 's have zero mean and unit variance) and $v_{i, k}$ denotes complex zero-mean white Gaussian noise with variance $\mathcal{N}_{0}$. The channel coefficients from Node $j$ to Node $k h_{j, k}, j=$ $0,1, \ldots, N, k=1,2 \ldots, N+1$, are complex Gaussian random variables with zero-mean and variances $\sigma_{j, k}^{2}$, where Node $(N+1)$ node is the destination node $d$. We assume coherence times of the channels are such that channel coefficients $h_{j, k}$ are not varying during $K$ consecutive time slots.

For the first $u$ phases, where $u$ depends on the protocol type, the non-cooperative transmission described above is repeated from the $n$th relay, $1 \leq n<u$ for retransmission of the source data. For the next phases, cooperative transmission is used for routing the source data toward the destination. In contrast to [3] where transmitters are able to adjust their phases, in this paper, the instantaneous CSI is not known at the transmitter nodes. This assumption is realistic for most wireless systems. Therefore, space-time codes are the appropriate choice to achieve spatial diversity gains. In Phase $n, u<n$, the previous $m$ nodes, where $m$ depends on the protocol type, transmit their signals simultaneously toward the next node using the appropriate space-time code structure, with a simple power allocation. The received signal at the $n$th receiving node in Phase $n$ is $\boldsymbol{y}_{n}=\left[y_{n, 1}, \ldots, y_{n, T}\right]^{t}$, which is given by

$$
\boldsymbol{y}_{n}=\boldsymbol{S}_{n} \boldsymbol{\Lambda}_{n} \boldsymbol{h}_{n}+\boldsymbol{v}_{n},
$$

for $n>u$, where $\boldsymbol{h}_{n}=\left[h_{n-m, n}, \ldots, h_{n-1, n}\right]^{t}, \boldsymbol{\Lambda}_{n}=$ $\operatorname{diag}\left[P_{n-m, n}, \ldots, P_{n-1, n}\right]$, and $P_{n-i, n}, i=1, \ldots, m$, is the average transmit power of node $n-i$ during the $i$ th time slot of Phase $n$. In (2), $\boldsymbol{S}_{n}$ is an appropriate $T \times m$ distributed space-time code.

It is assumed that all hops use the total bandwidth $W$. The objective of the system is the reliable delivery of symbols generated at the source node $s$ at a bandwidth-normalized rate (henceforth just called the rate of $R$ bits per second per Hertz, i.e., $R W$ bits per second) to the destination node by consuming the least total transmit power. Since the throughput is a continuous monotonously-increasing function of the transmission power, the throughput at all the hops to be equal to the desired one, i.e., $R$. Thus, to achieve the end-to-end rate $R$, it is obvious that all hops should guarantee the rate $R$.

In the sequel, the outage probability $\rho_{n-1, n} \triangleq \operatorname{Pr}\left\{r_{n}<\right.$ $R\}$ of the $n$th receiving node in hop $n$ is investigated, which describes the probability that the transmit rate $R$ is greater than the supported rate $r_{n}$. This probability expressed as a cumulative distribution function (CDF) and depends on the fixed transmission parameters and the channel condition within the hops. By defining $\gamma_{\text {th }} \triangleq\left(2^{R(N+1)}-1\right) \mathcal{N}_{0} W$, the outage probability of non-cooperative transmission can be represented as

$$
\rho_{n-1, n}=\operatorname{Pr}\left\{\sum_{i=1}^{2} P_{n-i, n}\left|h_{n-i, n}\right|^{2}<\gamma_{\mathrm{th}}\right\},
$$


for $n=1,2 \ldots, N+1$, where $P_{-1,1}=0$.

\section{TRANSMISSION PROTOCOLS}

In [9], the Alamouti code [11] is used for retransmitting the source data. In this section, we propose some efficient protocols for multihop cooperative routing employing distributed space-time codes of more than two cooperating nodes. The proposed protocols compromise between energy efficiency and rate using space-time coded cooperation and non-cooperative transmission. A general cooperation scenario, $m$-cooperation, $(1 \leq m \leq N)$, can be implemented in which each relay combines the signals received from the previous relays and along with that received from the source. For a general scheme $m$-cooperation, $(1 \leq m \leq N)$, each receiving node decodes the information after combining the signals received from the previous $m$ transmitting nodes.

\section{A. Broadcast-then-Cooperate Protocol}

In the first phase of transmission, the source sends $m$ symbols $s_{i}, i=1, \ldots, m$, which is received by $m-1$ nodes, which are closer to the source nodes.

Using [12, Eq. (8)], the appropriate source transmit power to guarantee the rate of $R$ can be found as

$$
P_{0,1}=\max \left\{\frac{-\gamma_{\mathrm{th}}}{\sigma_{0,1}^{2} \ln \left(1-\rho_{0}\right)}, \ldots, \frac{-\gamma_{\mathrm{th}}}{\sigma_{0, m-1}^{2} \ln \left(1-\rho_{0}\right)}\right\} .
$$

which can be simplified as

$$
P_{0,1}=\frac{-\gamma_{\mathrm{th}}}{\sigma_{\min , m}^{2} \ln \left(1-\rho_{0}\right)},
$$

where $\rho_{0}$ is the target outage probability at each hop, $\sigma_{\min , m}^{2}=$ $\min \left\{\sigma_{0,1}^{2}, \ldots, \sigma_{0, m-1}^{2}\right\}$, and $\gamma_{\mathrm{th}} \triangleq\left(2^{R(N-m+3)}-1\right) \mathcal{N}_{0} W$.

In Phase $p, p=2, \ldots, N-m+3$, nodes transmit space-time coded symbols using a $T \times m$ space-time code.

If we use full-rate space-times codes, in which $T=K$, such as distributed GABBA codes [12], the rate $r_{1}$ achieved at the first hop is

$$
r_{1}=\frac{1}{N-m+3} \log \left(1+\frac{P_{0,1}\left|h_{\min , m}\right|^{2}}{\mathcal{N}_{0} W}\right),
$$

where $\left|h_{\min , m}\right|^{2}=\min \left\{\left|h_{0,1}\right|^{2},\left|h_{0,2}\right|^{2}, \ldots,\left|h_{0, m-1}\right|^{2}\right\}$. which $T=K$, such as distributed GABBA codes [12], from (2) the rate $r_{n}$ achieved at Phase $p=2, \ldots, N-m+3$ is

$$
r_{p}=\frac{1}{N-m+3} \log \left(1+\sum_{i=1}^{m} \frac{P_{n-i, n}\left|h_{n-i, n}\right|^{2}}{\mathcal{N}_{0} W}\right),
$$

where the index of the $n$th relay can be represented in terms of Phase $p$ as $n=p+m-2$ and it is assumed that $\hat{s}_{n-i, k}=s_{k}$, $i=1, \ldots, m$ and $k=1, \ldots, K$.

In the case where $T>K$, the normalized rates in (6) and (7) can be rewritten as

$$
r_{1}=\frac{K}{K+T(N-m+2)} \log \left(1+\frac{P_{0,1}\left|h_{\min , m}\right|^{2}}{\mathcal{N}_{0} W}\right),
$$

$$
r_{p}=\frac{T}{K+T(N-m+2)} \log \left(1+\sum_{i=1}^{m} \frac{P_{n-i, n}\left|h_{n-i, n}\right|^{2}}{\mathcal{N}_{0} W}\right),
$$

for $p=2, \ldots, N-m+3$.

\section{B. Multihop-then-Cooperate Protocol}

In the previous subsection, the broadcast transmission from the source is used in the first phase of transmission. As it will be shown in the numerical results, for the case of a line network, non-cooperative transmission consumes less energy than the transmission protocol described in Subsection III-A. Here, we propose an alternative protocol that can be more power-efficient than the one in the previous section depending on the location of nodes - at the expense of a lower spectral efficiency.

In the first $(m-1)$ phases, non-cooperative multihop transmission is used for the communication of $(m-1)$ relays in proximity to the source node. The remaining transmission phases, i.e., Phase $n, n=m, \ldots, N+1$, is same as the one explained above. That is a $T \times m$ space-time code is used for the cooperative phases. Therefore, similar to noncooperative multihop transmission, we will have $N+1$ phases for transmission of $m$ symbols from the source to the destination. If we use full-rate space-times codes, in which $T=K$, such as distributed GABBA codes [12], we can achieve the same spectral efficiency as the protocol described in [9] and beamformer-based cooperative routing in [3]. That is, $N+1$ time slots per channel use. The rate achieved at the first $(m-1)$ phases is

$$
r_{n}=\frac{1}{N+1} \log \left(1+\frac{P_{n-1, n}\left|h_{n-1, n}\right|^{2}}{\mathcal{N}_{0} W}\right),
$$

for $n=1, \ldots, m-1$, where $P_{p-1, p}$ is the power transmitted from $p-1$ th node to the $p$ th node. The rate $r_{n}$ achieved at Phase $n=m, \ldots, N+1$ can be shown as

$$
r_{n}=\frac{1}{N+1} \log \left(1+\sum_{i=1}^{m} \frac{P_{n-i, n}\left|h_{n-i, n}\right|^{2}}{\mathcal{N}_{0} W}\right) .
$$

In the case that $T>K$, the normalized rates in (10) and (11) can be rewritten as

$$
\begin{gathered}
r_{n}=\frac{K}{K+T N} \log \left(1+\frac{P_{n-1, n}\left|h_{n-1, n}\right|^{2}}{\mathcal{N}_{0} W}\right), \\
r_{n}=\frac{T}{K+T N} \log \left(1+\sum_{i=1}^{m} \frac{P_{n-i, n}\left|h_{n-i, n}\right|^{2}}{\mathcal{N}_{0} W}\right),
\end{gathered}
$$

respectively.

\section{Full-Cooperation Protocol}

In this protocol, instead of using a single $T \times m$ dimensional space-time code, multiple space-time codes in different phases are employed. The cooperation protocol has $N+1$ phases, and consists of the following steps:

- The $m$ symbols are transmitted by the source terminal during the first and second time slots of Phase 1. In the first phase, the source node broadcasts its packet toward 
the relays and the destination. Thus, relays can estimate their source-to-relay channels.

- In Phase 2, relay nodes are sorted based on their received SNR, such that Relay 1 has the highest received SNR. Then, a group of $\left\lceil\frac{m}{2}\right\rceil$ Alamouti space-time codes, where $[\cdot]$ is the closest integer not lower than the argument, are used by the source and Relay 1 to retransmit those two packets. If $\frac{m}{2}$ is not an integer number, a column of one of the space-time codes sets to zero.

- In Phase $n, 2<n \leq N+1$, the previous $q_{n}=$ $\min \{m, n\}$ nodes transmit their signals toward the next node using a group of $T_{n} \times q_{n}$ space-time codes, with a simple power allocation. Assume that $k_{n}$ is the number of symbols in the space-time code. In this case, the number of space-time codes used for the retransmission of the source symbols is $\left[\frac{K}{k_{n}}\right]$. Thus, $\left.\mid \frac{K}{k_{n}}\right\rfloor$ space-time code of size $T_{n} \times q_{n}$ are employed for the retransmission of most of symbols. For the remaining part of the symbols a space-time code with $\bmod \left(K, k_{n}\right)$ symbols and $\bmod \left(m, q_{n}\right)$ columns is employed.

For constructing the full-rate, full-diversity $T_{n} \times q$ space-time codes, one can delete columns of $N \times N$ GABBA space-time codes, where $N$ is a power of two.

If full-rate space-times codes are used, in which $T=K$ and $T_{n}=k_{n}, n=2, \ldots, N+1$, such as distributed GABBA codes [12], the rate we can achieve has the same spectral efficiency as the protocol described in [9], that is, $N+1$ time slots per channel use. The rate achieved at the $n$th phase is

$$
r_{n}=\frac{1}{N+1} \log \left(1+\sum_{i=1}^{q_{n}} \frac{P_{n-i, n}\left|h_{n-i, n}\right|^{2}}{\mathcal{N}_{0} W}\right)
$$

for $n=1, \ldots, N+1$, where $q_{n}=\min \{m, n\}$. In the case that $T>K$, the normalized rates in (14) can be rewritten as

$$
r_{n}=\frac{T_{n}}{\sum_{i=1}^{N+1} T_{i}} \log \left(1+\sum_{i=1}^{m} \frac{P_{n-i, n}\left|h_{n-i, n}\right|^{2}}{\mathcal{N}_{0} W}\right)
$$

for $n=1, \ldots, N+1$, where $T_{1}=K$.

\section{Distributed Per-Hop Outage Constrained POWER Allocation}

In this section, we derive the required power for the direct (non-cooperative) and cooperative transmission modes in order to achieve a certain rate $R$.

In the non-cooperative case, only one node is transmitting within a time slot to a single receiving node. The transmitter node should decide the value of its transmit power $P_{n-1, n}$ to satisfy the target rate $R$ with a target outage probability of $\rho_{0}$ at each hop. The per-hop outage probability constraint is used for distributed power allocation. The receiver can correctly decode the source data whenever $P_{n-i, n}\left|h_{n-i, n}\right|^{2} \geq \gamma_{\text {th }}$. Now, assume a connection from the source node to the destination via $N$ intermediate nodes. For decoding the message reliably, the outage probability must be less than a desired end-to-end outage probability $\rho_{\max }$. Thus, in the multihop case, a target outage probability $\rho_{0}=1-\sqrt[N+1]{1-\rho_{\max }}$ is required at each hop. Using (5), the total power per symbol is given by

$P_{T}($ non-coop $)=\sum_{n=1}^{N+1} \mathcal{C}(n-1, n)=\sum_{n=1}^{N+1} \frac{-\gamma_{\text {th }}(N+1)}{\sigma_{n-1, n}^{2} \ln \left(1-\rho_{\max }\right)}$,

where $\mathcal{C}(n-1, n)$ is the point-to-point link cost when the $(n-$ $1)$ th node transmits to the $n$th node.

Now, we consider the cooperative link cost formulation. In this case, from (5) the source node transmits with the power $P_{0,1}=\frac{-\gamma_{\text {th }}}{\sigma_{x}^{2} \ln \left(1-\rho_{0}\right)}$ during the first phase, where $\sigma_{x}^{2}=\sigma_{0,1}^{2}$ for Multihop-then-Cooperate (MTC) and Full-Cooperation (FC) protocols, and $\sigma_{x}^{2}=\sigma_{\min , m}^{2}$ for Broadcast-then-Cooperate (BTC) protocol. For Broadcast-then-Cooperate protocol, from (6) and (7), we have $\gamma_{\text {th }}=\left(2^{R(N-m+3)}-1\right) \mathcal{N}_{0} W$. For Multihop-then-Cooperate and Full-Cooperation protocols, we have $\gamma_{\text {th }}=\left(2^{R(N+1)}-1\right) \mathcal{N}_{0} W$. In Phase $n$, a set of $m$ nodes $\mathrm{Tx}_{n}=\left\{\mathrm{tx}_{n, 1}, \ldots, \mathrm{tx}_{n, m}\right\}$ cooperate to transmit the source's information to a single receiver node $\mathrm{rx}_{n}$, using the $T \times m$ space-time code, as stated in (2). For coherent detection at the receiving node, the signals simply add up at the receiver, and acceptable decoding is possible when $\gamma_{n}^{\text {rec }}=\sum_{i=1}^{m} P_{n-i, n}\left|h_{n-i, n}\right|^{2} \geq \gamma_{\text {th }}$. Our objective is to find the minimum value of the total transmission power in Phase $n$, i.e., the cost function $\mathcal{C}\left(\mathrm{Tx}_{n}, n\right)=\sum_{i=1}^{m} P_{n-i, n}$, such that the outage probability at the receiving node $\mathrm{rx}_{n}$ becomes less than the target value $\rho_{0}$.

Now, a tractable outage probability formula is derived for the sum of independent-not-identical exponentially distributed random variables at the receiving node $\mathrm{rx}_{n}$. The moment generating function (MGF) of the random variable $\gamma_{n}^{\text {rec }}$ is derived to calculate $\operatorname{Pr}\left\{\gamma_{n}^{\text {rec }}<\gamma_{\text {th }}\right\}$. Since the $\gamma_{i}$ 's are independent exponential random variables, the MGF of $\gamma_{n}^{\text {rec }}$, i.e., $M_{n}(-s)=\mathbb{E}\left\{e^{-s \gamma_{n}^{\text {rec }}}\right\}$, can be written as $M_{n}(-s)=$ $\prod_{i=1}^{m} \frac{1}{1+P_{n-i, n} \sigma_{n-i, n}^{2} s}$. Using partial fraction expansion, and by assuming that the products $P_{n-i, n} \sigma_{n-i, n}^{2}$ are distinct for all links, the MGF can be decomposed into

where

$$
M_{n}(-s)=\sum_{i=1}^{m} \frac{\alpha_{m, n, i}}{1+P_{n-i, n} \sigma_{n-i, n}^{2} s},
$$

$$
\alpha_{m, n, i}=\prod_{\substack{j=1 \\ j \neq i}}^{m} \frac{P_{n-i, n} \sigma_{n-i, n}^{2}}{P_{n-i, n} \sigma_{n-i, n}^{2}-P_{n-j, n} \sigma_{n-j, n}^{2}} .
$$

Since each term in the summation in (17) corresponds to the MGF of an exponential distribution, $\operatorname{Pr}\left\{\gamma_{n}^{\text {rec }}<\gamma_{\text {th }}\right\}$ can be written as

$$
\begin{aligned}
\rho_{n, n-1} & =\operatorname{Pr}\left\{\gamma_{n}^{\mathrm{rec}}<\gamma_{\mathrm{th}}\right\} \\
& =\sum_{i=1}^{m} \alpha_{m, n, i}\left(1-e^{\frac{-\gamma_{\mathrm{th}}}{P_{n-i, n} \sigma_{n-i, n}^{2}}}\right)
\end{aligned}
$$

Further, we formulate the problem of power allocation in cooperative multihop networks. The link cost or total transmitted power for the multipoint-to-point case is $\mathcal{C}\left(\operatorname{Tx}_{n}, n\right)=$ $\sum_{i=1}^{m} P_{n-i, n}$. Therefore, the power allocation problem, which has a required outage probability constraint on the receiving node, can be formulated as 


$$
\begin{aligned}
\min & \sum_{i=1}^{m} P_{n-i, n}, \\
\text { s.t. } & \sum_{i=1}^{m} \alpha_{m, n, i}\left(1-e^{\frac{-\gamma_{\text {th }}}{P_{n-i, n} \sigma_{n-i, n}^{2}}}\right) \leq \rho_{0} . \\
& P_{n-i, n} \geq 0, \text { for } i=1, \ldots, m .
\end{aligned}
$$

The constraint function in (20) is not convex. For tractability, the outage probability in (19) is rewritten in terms of its series representation as

$$
\rho_{n-1, n}=\sum_{i=1}^{m} \alpha_{m, n, i} \sum_{k=1}^{\infty} \frac{1}{k !}\left(\frac{-\gamma_{\mathrm{th}}}{P_{n-i, n} \sigma_{n-i, n}^{2}}\right)^{k}
$$

and by replacing $\alpha_{m, n, i}$ from (18) into (21), $\rho_{n-1, n}$ can be well approximated in high SNR as

$$
\rho_{n-1, n} \leq \frac{\gamma_{\mathrm{th}}^{m}}{m ! \prod_{i=1}^{m} \sigma_{n-i, n}^{2} P_{n-i, n}}
$$

where we have used the fact that $\sum_{i=1}^{m} \frac{\alpha_{m, n, i}}{P_{n-i, n}^{k} \sigma_{n-i, n}^{2 k}}=0$, $k=1, \ldots, m-1$, for deriving (22). The expression in (22) is a tight upper-bound on the outage probability, and thus, it can be reliably used for power allocation along nodes. The optimization problem [9, Eq. (18)] in which two nodes cooperatively transmit in Phase $n$ can be modified for $m$ cooperative transmission as

$$
\begin{array}{ll}
\min & \sum_{i=1}^{m} P_{n-i, n}, \\
\text { s.t. } & \frac{\gamma_{\mathrm{th}}^{m}}{m ! \prod_{i=1}^{m} \sigma_{n-i, n}^{2} P_{n-i, n}} \leq \rho_{0}, \\
& P_{n-i, n} \geq 0, \text { for } i=1, \ldots, m .
\end{array}
$$

The optimal power allocation strategy for high SNRs is found in the following theorem. Since the approximate outage probability expression derived in (22) is an upper-bound on the outage probability, this result can be used reliably for all SNR scenarios.

Theorem 1: The optimum power allocation $P_{n-i, n}^{*}$ and $i=$ $1, \ldots, m$ in the optimization problem stated in (23) are equal and can be expressed as

$$
P_{n-i, n}^{*}=\frac{\gamma_{\mathrm{th}}}{\sqrt[m]{m ! \rho_{0} \prod_{i=1}^{m} \sigma_{n-i, n}^{2}}}, i=1, \ldots, m
$$

Proof: Proof is similar to the proof of Theorem 1 of [9].

An interesting property of $P_{n-i, n}^{*}$ in (24) is that it is just dependent on the product of all path-loss coefficients of links. Therefore, $P_{n-i, n}^{*}$ s can be calculated in a decentralized manner by broadcasting the product term from the receiving node toward the transmitting nodes. Using Theorem 1 and (5), the total transmission power for the Broadcast-then-Cooperate protocol

$$
\begin{aligned}
& P_{T}(\mathrm{BTC})=\sum_{p=1}^{N-m+3} \mathcal{C}\left(\operatorname{Tx}_{p}, p\right)=P_{0,1}^{*}+\sum_{n=m}^{N+1} \sum_{i=1}^{m} P_{n-i, n}^{*} \\
& =\frac{-\gamma_{\mathrm{th}}}{\sigma_{\mathrm{min}, n}^{2} \ln \left(1-\rho_{0}\right)}+\sum_{n=m}^{N+1} \frac{m \gamma_{\mathrm{th}}}{\sqrt[m]{m ! \rho_{0} \prod_{i=1}^{m} \sigma_{n-i, n}^{2}}}
\end{aligned}
$$

For the case of Multihop-then-Cooperate and Multihop-thenCooperate and Full-Cooperation protocols, the total power become

$$
\begin{aligned}
& P_{T}(\mathrm{MTC})=\sum_{n=1}^{N+1} \mathcal{C}\left(\mathrm{Tx}_{n}, n\right)=\sum_{n=1}^{m-1} P_{n-1, n}^{*}+\sum_{n=m}^{N+1} \sum_{i=1}^{m} P^{m} \\
& =\sum_{n=1}^{m-1} \frac{-\gamma_{\mathrm{th}}}{\sigma_{n-1, n}^{2} \ln \left(1-\rho_{0}\right)}+\sum_{n=m}^{N+1} \frac{m \gamma_{\mathrm{th}}}{\sqrt[m]{m ! \rho_{0} \prod_{i=1}^{m} \sigma_{n-i, n}^{2}}} \\
& P_{T}(\mathrm{FC})=\sum_{n=1}^{N+1} \mathcal{C}\left(\mathrm{Tx}_{n}, n\right) \\
& +\sum_{n=2}^{m-1} \frac{-\gamma_{\mathrm{th}}}{\sigma_{0,1}^{2} \ln \left(1-\rho_{0}\right)} \\
& \sqrt{q ! \rho_{0} \prod_{i=1}^{q} \sigma_{n-i, n}^{2}}
\end{aligned}
$$

The outage probability $\rho_{n}$ at the $n$th receiver is affected by all previous $n$ nodes and can be iteratively calculated according to the recursion

$$
\rho_{n}(m) \leq 1-\left(1-\rho_{n-1, n}(m)\right) \prod_{i=\max \{1, n-m\}}^{n-1}\left(1-\rho_{i}(m)\right),
$$

for $n=1, \ldots, N+1$, when MTC protocol is used. This formula can be also used for two other proposed protocols. In the case of BTC and FC protocols, (28) is valid for $n=m+1, \ldots, N+1$, and $\rho_{j}(m) \leq \rho_{0}$, for $j=1, \ldots, m-1$.

It is important to note that assuming the equality in (28) implies that the error at the destination occurs even if one intermediate node experience an error. This guarantees that by using the power allocation strategies given in Theorem 1, the outage probability QoS at the destination is satisfied.

$\rho_{\text {error }}(m)=\rho_{N+1}(m)=1-\prod_{\nu=0}^{N}\left(1-\rho_{N-\nu, N-\nu+1}(q)\right)^{\Omega_{m}(\nu)}$,

where for the case of FC protocol, we have $\Omega_{m}(-1)=0$, $\Omega_{m}(0)=1$, and $\Omega_{m}(\nu)=\sum_{i=1}^{\min }\{m, \nu\} \Omega_{m}(\nu-i)$. Note that for the case of $m=2,\left\{\Omega_{2}(\nu)\right\}$ is a Fibonacci sequence, i.e., $\Omega_{2}(\nu)=\Omega_{2}(\nu-1)+\Omega_{2}(\nu-2)$. Also, for the extreme case of $m=N+1$, we have $\Omega_{N+1}(\nu)=2^{\nu-1}$. When BTC and MTC protocol is used, we have $\Omega_{m}(\nu)=1,0 \leq \nu<m$.

To get an insight into the relationship between the end-toend probability of error $\rho_{\max }=\rho_{N+1}$ and $\rho_{0}$, we have

$\rho_{\max }=1-\prod_{\nu=0}^{N}\left(1-\rho_{0}\right)^{\Omega_{m}(\nu)}=1-\left(1-\rho_{0}\right)^{\sum_{\nu=0}^{N} \Omega_{m}(\nu)}$. 


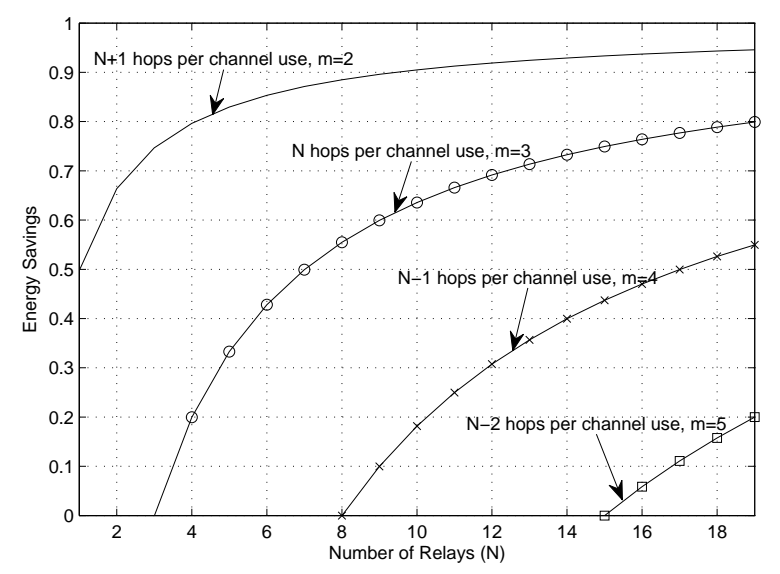

Fig. 1. The average energy savings curves versus the number of relays, using the distributed protocols for cooperative routing with distributed spacetime codes of $m$ cooperating nodes when the end-to-end outage probability is $\rho_{\max }=10^{-4}$.

for FC and MTC protocols, and

$$
\rho_{\max } \leq 1-\prod_{\nu=m}^{N}\left(1-\rho_{0}\right)^{\Omega_{m}(\nu)}=1-\left(1-\rho_{0}\right)^{\sum_{\nu=m}^{N} \Omega_{m}(\nu)}
$$

for the BTC protocol. Thus, the target outage probability at each hop $\rho_{0}$ can be represented in terms of the end-to-end desired probability of error $\rho_{\max }$.

\section{NUMERICAL ANALYSIS}

In this section, numerical results are provided to quantify the energy savings (see [9, Eq. (29)]) using the proposed cooperative routing protocols. A regular line topology is considered where nodes are located at unit distance from each other on a straight line. The optimal non-cooperative routing in this network is to always send the information to the next nearest node in the direction of the destination. Assume that the noise power $\mathcal{N}_{0}$, rate $R$, and bandwidth $W$ are normalized to 1 .

In Fig. 1, we compare the achieved energy savings of the proposed outage-restricted cooperative routings with respect to the non-cooperative multihop scenario. We compare the distributed power allocation protocols derived in Section III. Here, $\sigma_{i, j}^{2}$ is proportional to the inverse of the squared distance, i.e., $\sigma_{i, j}^{2}=\frac{1}{d^{\nu}}$, where it is assumed $\nu=3$, and the end-toend outage probability is $\rho_{\max }=10^{-4}$. It can be observed from Fig. 1 that using the full-cooperation power control, around $55 \%$ saving in energy are achieved when 3 relays are employed. It is shown that FC cooperative routing is beneficial for networks with small number of relays. In addition, it is shown that using MTC and BTC protocols, substantial savings in energy are achievable when the number of relays is close to the cooperation factor $m$.

Fig. 2 shows the average energy savings curves of BTC protocol versus the number of relays for the per-hop outage probability of $\rho_{0}=10^{-5}$ and different values for $m$. As it

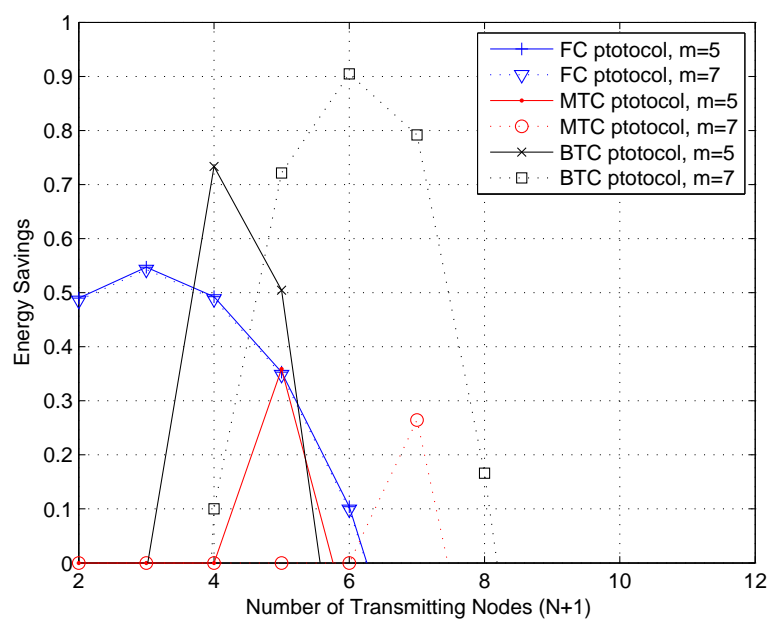

Fig. 2. The average energy savings curves versus the number of relays, using the distributed power allocation for cooperative routing with distributed space-time codes of $m$ cooperating nodes when per hop outage probability requirement is $\rho_{0}=10^{-5}$.

can be seen, for the assumed Line Network, a system with lower cooperation factor $m$ performs better in terms of energy savings, at the expense of lower spectral efficiency.

\section{REFERENCES}

[1] N. Vaidya, "Open problems in mobile ad-hoc networks," in Keynote talk at the Workshop on Local Area Networks, (Tampa, Florida), Nov. 2001.

[2] F. Li, K. Wu, and A. Lippman, "Energy-efficient cooperative routing in multi-hop wireless ad hoc networks," in IEEE International Performance, Computing, and Communications Conference, pp. 215-222, Apr. 2006.

[3] A. E. Khandani, J. Abounadi, E. Modiano, and L. Zheng, "Cooperative routing in static wireless networks," IEEE Trans. Commun., vol. 55, no. 11, pp. 2185-2192, Nov. 2007.

[4] A. S. Ibrahim, Z. Han, and K. J. R. Liu, "Distributed energy-efficient cooperative routing in wireless networks," IEEE Trans. Wireless Commun., vol. 7, no. 11, pp. 757-759, Oct. 2008.

[5] C. Pandana, W. P. Siriwongpairat, T. Himsoon, and K. J. R. Liu, "Distributed cooperative routing algorithms for maximizing network lifetime," in IEEE Wireless Communications and Networking Conference (WCNC), vol. 1, pp. 451-456, Apr. 2006.

[6] Z. Yang and A. Host-Madsen, "Routing and power allocation in asynchronous Gaussian multiple-relay channels," EURASIP J. Wireless Commun. and Networking, 2006.

[7] B. Maham and A. Hjørungnes, "Near-optimum power allocation for ber restricted multihop cooperative networks," in Proc. IEEE International Conference on Communications (ICC'10), (Cape Town, South Africa), May 2010.

[8] W. Saad, Z. Han, M. Debbah, A. Hjørungnes, and T. Basar, "A gamebased self-organizing uplink tree for VoIP services in IEEE 802.16j networks," in IEEE International Conference on Communications (ICC), (Dresden, Germany), June 2009.

[9] B. Maham, R. Narasimhan, and A. Hjørungnes, "Energy-efficient spacetime coded cooperative routing in multihop wireless networks," in IEEE GLOBECOM, (Honolulu, Hawaii, USA), Nov.- Dec. 2009.

[10] R. Mudumbai, D. R. B. III, U. Madhow, and H. V. Poor, "Distributed transmit beamforming: Challenges and recent progress," IEEE Cотmunications Magazine, vol. 47, no. 2, pp. 102-110, Feb. 2009.

[11] S. M. Alamouti, "A simple transmitter diversity scheme for wireless communications," IEEE Journal on Selected Areas in Communications, vol. 16, no. 8, pp. 1451-1458, Oct. 1998.

[12] B. Maham, A. Hjørungnes, and G. Abreu, "Distributed GABBA spacetime codes in amplify-and-forward relay networks," IEEE Trans. Wireless Commun., vol. 8, no. 4, pp. 2036-2045, Apr. 2009. 